\title{
Self-trapped magnetic polaron: Exact solution of a continuum model in one dimension
}

\author{
S. Pathak and S. Satpathy \\ Department of Physics and Astronomy, University of Missouri, Columbia, Missouri 65211
}

(Received 1 June 2000; published 9 May 2001)

\begin{abstract}
A continuum model for the self-trapped magnetic polaron is formulated and solved in one dimension using a variational technique as well as the Euler-Lagrange method, in the limit of $J_{H} \rightarrow \infty$, where $J_{H}$ is the Hund'srule coupling between the itinerant electron and the localized lattice spins treated as classical spins. The Euler-Lagrange equations are solved numerically. The magnetic polaron state is determined by a competition between the electron kinetic energy, characterized by the hopping integral $t$, and the energy of the antiferromagnetic lattice, characterized by the exchange integral $J$. In the broad-band case, i.e., for large values of $\alpha$ $\equiv t / J S^{2}$, the electron nucleates a saturated ferromagnetic core region (type-II polaron) similar to the Mott description, while in the opposite limit, the ferromagnetic core is only partially saturated (type-I polaron), with the crossover being at $\alpha_{c} \approx 7.5$. The magnetic polaron is found to be self-trapped for all values of $\alpha$. The continuum results are also compared to the results for the discrete lattice.
\end{abstract}

DOI: 10.1103/PhysRevB.63.214413

\section{INTRODUCTION}

A self-trapped magnetic polaron is formed when an itinerant carrier nucleates a ferromagnetic core region in an otherwise antiferromagnetic lattice of localized spins and traps itself in the core. ${ }^{1-7}$ The energy cost to form the ferromagnetic core is balanced by the energy gain of the itinerant carrier. This object has been given the name "ferron" by Nagaev. ${ }^{8}$ The carrier thus disrupts the antiferromagnetic order in the "self-trapped" region, but away from it the antiferromagnetic order is restored. The prototypical candidates for the self-trapped magnetic polaron are thought to be EuSe and EuTe, on which considerable attention has been focused in the past. ${ }^{9-13}$ Interest on the magnetic polaron problem has recently resurfaced in light of its relevance to a number of new systems, e.g., the high- $T_{c}$ materials and the colossal magnetoresistive manganites. ${ }^{14^{c}-19}$

In this paper, we formulate a continuum model and solve it exactly using the Euler-Lagrange method and study the energetics and the spin structure of the magnetic polaron. The Euler-Lagrange equations are solved numerically using an iterative procedure that yields "exact" results in the sense that the solution can be obtained to any arbitrary accuracy. The results are compared with the Mott picture as well as with the results of a variational method, where energy is minimized by varying the parameters of a trial wave function. One key result is that the magnetic polaron is found to be self-trapped for all values of the electronic parameter $\alpha$ $\equiv t / J S^{2}$ in contrast to the Mott picture, but in agreement with de Gennes's results for the case of the lattice, where the magnetic polaron was found to be self-trapped for all values of $\alpha{ }^{1}$

\section{HAMILTONIAN}

We consider the following Hamiltonian describing the magnetic polaron in a lattice:

$\mathcal{H}=-t \sum_{\langle i j\rangle, \sigma} a_{i \sigma}^{\dagger} a_{j \sigma}+$ H.c. $-J_{H} \sum_{i} \vec{s}_{i} \cdot \vec{S}_{i}+J \sum_{\langle i j\rangle} \vec{S}_{i} \cdot \vec{S}_{j}$
PACS number(s): 75.25.+z, 75.90.+w a model studied by several authors in its lattice version. $^{3-5,8,19}$ The three terms in the Hamiltonian describe, respectively, the kinetic energy of the itinerant electron, the Hund's-rule coupling between the electron and the localized spin on each site on the lattice, and the antiferromagnetic exchange between the localized spins. The symbols $a_{i \sigma}^{\dagger}$ and $a_{i \sigma}$ denote the creation and the annihilation operators for the electron state with spin $\sigma$ at site $i,\langle i j\rangle$ denotes summation over nearest-neighbor sites, $s_{i}$ is the spin of the itinerant electron, and $S_{i}$ denotes the spin of the localized electron, the latter treated as classical spins. Note that in the onedimensional case with nearest-neighbor hopping, itinerant electron transfer is allowed only between lattice sites belonging to the two different magnetic sublattices. Second and higher nearest-neighbor transfers could adversely affect the stability of the magnetic polaron ${ }^{4}$ and so could the quantum fluctuations if the localized spins are treated as quantum spins instead of classical spins.

The Hamiltonian parameters $t, J_{H}$, and $J$ are all taken to be positive. In this work we shall take $J_{H} \rightarrow \infty$ as appropriate for many solids including the manganites. Note that a positive $J$, leading to an antiferromagnetic spin structure in the absence of any charge carriers, is crucial to the description of the problem. In the context of the manganites, band calculation $\mathrm{s}^{20}$ as well as traditional wisdom show the itinerant electron to come from the $\operatorname{Mn}\left(e_{g}\right)$ states, while the $\operatorname{Mn}\left(t_{2 g}\right)$ electrons constitute the localized spins, interacting antiferromagnetically. The typical parameters in the solids are $t \approx 0.1 \mathrm{eV}$ and $J S^{2} \approx 10 \mathrm{meV}$, so that the dimensionless parameter $\alpha \equiv t / J S^{2} \approx 10$.

\section{MOTT POLARON}

The simplest description of the magnetic polaron is due to Mott. ${ }^{5}$ Mott considered a self-trapped state, where the selftrapped region of radius $R$ is fully ferromagnetic and the region outside it is fully antiferromagnetic (this scenario is sketched in Fig. 1). The size of the self-trapped region in one dimension is obtained by minimizing the Mott total energy,

$$
E_{M o t t}=-2 t+\hbar^{2} \pi^{2} /\left(8 m R^{2}\right)+2 R\left(2 J S^{2}\right)-J_{H} S .
$$




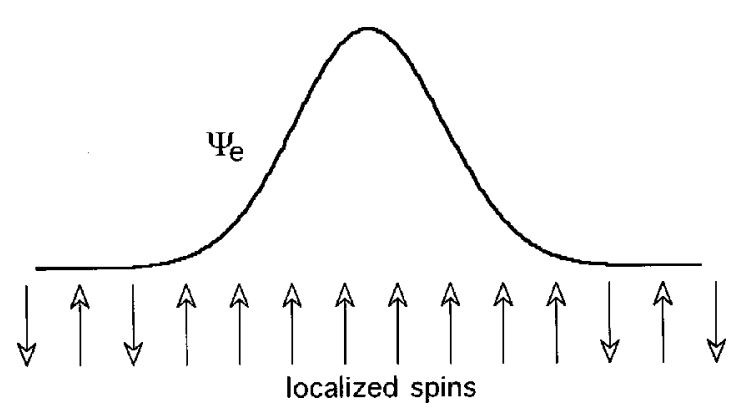

FIG. 1. Sketch of the Mott picture of the self-trapped magnetic polaron, indicating the disruption of the antiferromagnetic order and the creation of a ferromagnetic region, where the charge carrier becomes self-trapped. Arrows indicate the localized spins, while the line shows the wave function of the trapped electron.

The first two terms here describe the electronic kinetic energy, with the quantity $-2 t$ being simply the band structure energy of the propagating state with Bloch momentum $k$ $=0$ and the second term being the confinement energy of the electron (particle in a box). The third term is the exchange energy cost to align the localized spins ferromagnetically inside the self-trapped region. Finally, the last term is the energy gain due to the Hund's-rule coupling between the itinerant electron and the localized spins.

To determine the stability of the self-trapped magnetic polaron, one has to compare the polaron energy with that of the propagating electron state, which for the antiferromagnetic spin lattice can be easily shown to be $E_{k}$ $= \pm \sqrt{J_{H}^{2} S^{2}+4 t^{2} \cos ^{2} k a}, k$ being the Bloch momentum and $a$ being the lattice constant. The propagating state with a ferromagnetic lattice is unfavorable because of the high exchange energy cost for the localized spins.

Taking $J_{H} \rightarrow \infty$ and using the relation in the tight-binding band theory $\hbar^{2} /\left(2 t a^{2}\right)=m$, the following results are obtained for the Mott polaron in one dimension: (a) The energy of the polaron $E / t=3(\pi / \alpha)^{2 / 3}-2$; (b) a localized (selftrapped) magnetic polaron forms for the dimensionless parameter $\alpha \equiv t / J S^{2}>\alpha_{c}$, where $\alpha_{c}=\left(27 \pi^{2} / 8\right)^{1 / 2} \sim 5.8$, beyond which the propagating state has the lower energy; and (c) the radius of the self-trapped polaron is given by $R_{\text {min }}$ $=\left(\pi^{2} \alpha / 8\right)^{1 / 3} a$. In contrast to Mott's result, de Gennes ${ }^{1}$ has argued that the polaron should be self-trapped for all values of $\alpha$ by considering a few-site discrete lattice model comprising the central site of the magnetic polaron and its nearest neighbors.

\section{WAVE FUNCTION}

Since we take $J_{H}=\infty$, the electron spin is always parallel to the localized spins as the electron moves about in the lattice. The electron-hopping amplitude is then given by the Anderson-Hasegawa result of $-t \cos (\chi / 2)$, where $\chi$ is the cant angle between two neighboring core spins. ${ }^{21}$ The state of the system is described by the orientation $\left(\theta_{n}, \phi_{n}\right)$ of the core spins at each lattice site together with the amplitudes of the electronic wave function,

$$
|\psi\rangle=\sum_{n} c_{n} a_{n}^{\dagger}\left(\theta_{n}, \phi_{n}\right)|0\rangle
$$

The operator $a_{n}^{\dagger}\left(\theta_{n}, \phi_{n}\right)$ creates an electron at the $n$th site with the electron spin oriented along the direction of the core spin at that site. If $J_{H}$ was finite, we would, of course, need to have a two-component spinor for the electron wave function at each site. Taking now the azimuthal angles $\phi_{n}$ to be zero (justified in general by the existence of the easy planes in the solid), the ground state of the system is obtained by minimizing the total energy

$$
\begin{aligned}
E= & -t \sum_{\langle n m\rangle} c_{n}^{*} c_{m} \cos \frac{\theta_{n}-\theta_{m}}{2}+\text { c.c. } \\
& +J S^{2} \sum_{\langle n m\rangle} \cos \left(\theta_{n}-\theta_{m}\right)-J_{H} S .
\end{aligned}
$$

\section{CONTINUUM MODEL}

We now take the continuum limit, where the amplitude of the electronic wave function and the cant angle between the neighboring core spins are taken as continuous variables: $c_{n} \rightarrow \sqrt{a} \psi(x)$ and $\theta_{n+1}-\theta_{n} \equiv \chi_{n} \rightarrow \chi(x)$. The continuum approximation is valid if the polaron radius is large as compared to the lattice constant. The total energy Eq. (4) may now be written as

$$
\begin{aligned}
E= & -t \int_{-\infty}^{\infty}\left[2 \psi^{2}(x)+\psi(x) \frac{d^{2} \psi}{d x^{2}}\right] \cos \frac{\chi(x)}{2} d x \\
& +J S^{2} \int_{-\infty}^{\infty}[\cos \chi(x)+1] d x,
\end{aligned}
$$

where we have expanded the electronic wave function $\psi(x)$ and the cant angle $\chi(x)$ in Taylor series. The first term in Eq. (5) is the kinetic energy of the electron, where the multiplicative $\cos (\chi / 2)$ factor comes from the AndersonHasegawa double exchange and the second term is the exchange energy of the core spins. The zero of energy has been redefined to be the energy of the lattice of the antiferromagnetic core spins. Notice that the energy is still zero even when an itinerant electron is present in the antiferromagnetic lattice because the antiferromagnetic core spins suppress the electron hopping due to the Anderson-Hasegawa $\cos (\chi / 2)$ factor. Also in Eq. (5) and in the rest of the paper, the unit of length is taken to be the lattice constant $a=1$.

\section{VARIATIONAL SOLUTION}

In our variational treatment, we obtain the ground state of the magnetic polaron by minimizing the total energy Eq. (5) with respect to the following variational wave function:

$$
\begin{gathered}
\psi(x)=N / \cosh (\lambda x), \\
\chi(x)= \begin{cases}2 \cos ^{-1}\left(\eta t \psi^{2} / 4 J S^{2}\right), & \text { if } \eta t \psi^{2} / 4 J S^{2} \leqslant 1 \\
0, & \text { otherwise. }\end{cases}
\end{gathered}
$$




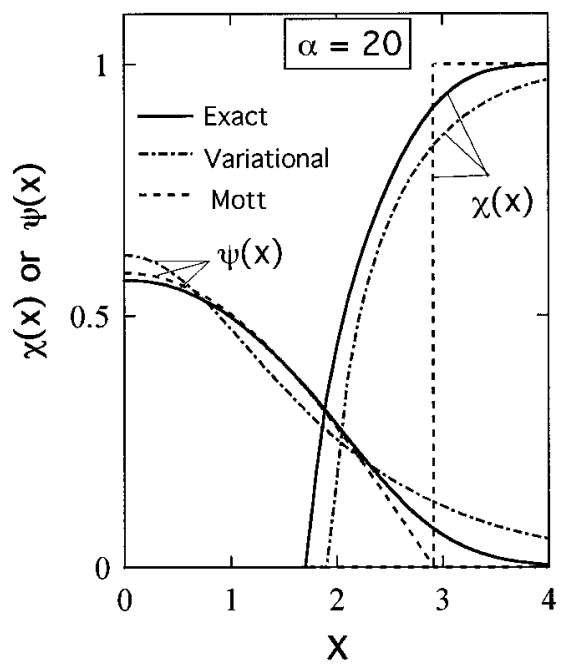

FIG. 2. The normalized electronic wave functions $\psi(x)$ and the cant-angle functions $\chi(x)$ calculated for the continuum model from the Mott, variational, and the exact (Euler-Lagrange) solutions for $\alpha=20$. The cant angles $\chi$ are in units of $\pi$, so that we have a ferromagnetic central region $(\chi=0)$ that turns into an antiferromagnetic region $(\chi=\pi)$ away from the center of the polaron as seen from the figure. All wave functions and cant angles are shown for positive $x$ only, since they are symmetric about the origin.

Here $\lambda$ and $\eta$ are the two variational parameters and $N$ is the normalization constant. The form of the electronic part $\psi(x)$ was chosen so that it behaves like a free particle at the center of the polaron $x \rightarrow 0$ and it decays exponentially at long distances $x \rightarrow \infty$.

The form of the cant angle $\chi(x)$ was chosen following the expression for the cant angle expected from the doubleexchange model. To see this, consider a system of two spins with an antiferromagnetic interaction $J$ and take the number of electrons $n$ mediating double exchange between them to be the local wave function squared $|\psi|^{2}$. The cant angle $\chi$ between the two spins is then obtained by minimizing the energy

$$
E=-t \psi(x)^{2} \cos (\chi / 2)+J S^{2} \cos \chi,
$$

which leads to the result

$$
\chi(x)=2 \cos ^{-1}\left[t \psi(x)^{2} / 4 J S^{2}\right] .
$$

From this expression, a ferromagnetic alignment $\chi=0$ is obtained for sufficiently large values of $n$. The variational wave function Eq. (6) reflects this form except that the variational parameter $\eta$ has been introduced. We note that $\eta$ is expected

TABLE I. Calculated ground-state energies and variational parameters $\lambda$ and $\eta$ for the trial wave function Eq. (6). Energies are in units of $t$.

\begin{tabular}{rccc}
\hline \hline$\alpha$ & Energy & $\lambda$ & $\eta$ \\
\hline 1 & -0.087 & 0.82 & 1.60 \\
10 & -0.885 & 0.79 & 1.78 \\
100 & -1.696 & 0.55 & 2.23 \\
\hline \hline
\end{tabular}
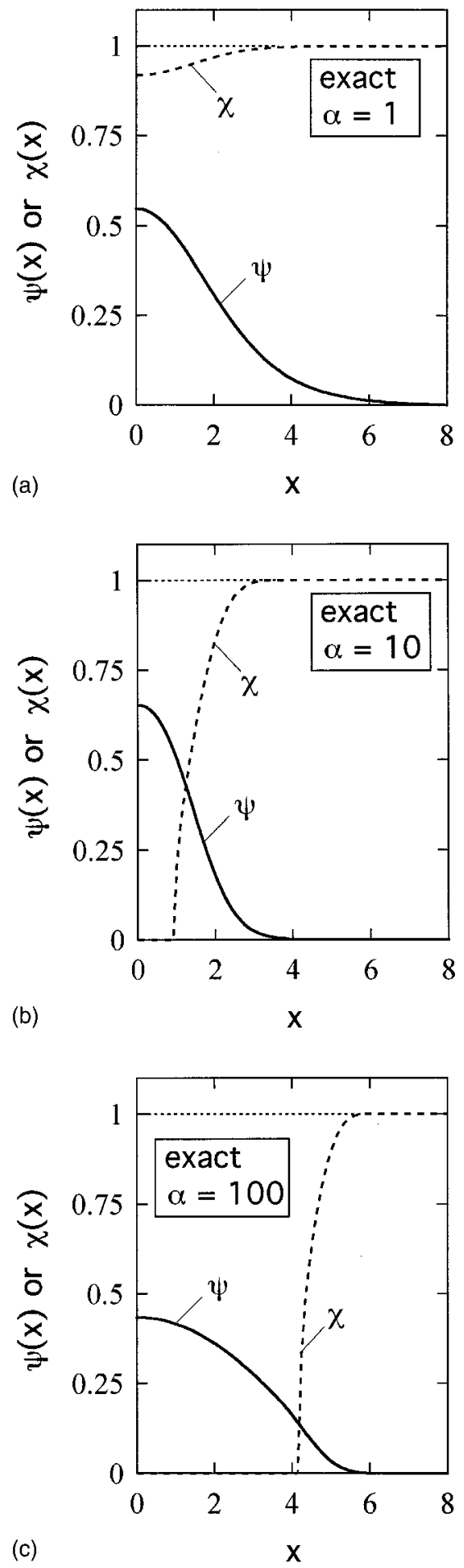

FIG. 3. The electronic wave function $\psi(x)$ and the cant-angle function $\chi(x)$ obtained from the exact calculation for the case $\alpha=1$ (a), 10 (b), and 100 (c).

to be of the order of the number of nearest neighbors in the solid (two in one dimension), since the kinetic energy term in Eq. (7) becomes multiplied by this factor. In addition to the trial function (6), we have in fact tried several others. Among those tried, the trial function Eq. (6) gives the best overall result in the range of $\alpha$ considered. The variational parameters were obtained numerically by minimizing the total en- 
TABLE II. Ground-state energies of the magnetic polaron for several values of $\alpha \equiv t /\left(J S^{2}\right)$. Energies are in units of $t .\left\langle x^{2}\right\rangle^{1 / 2}$ indicates the size of the self-trapped region in units of the lattice constant.

\begin{tabular}{rcccccc}
\hline \hline$\alpha$ & $\begin{array}{c}\text { Energy } \\
\text { Mott }\end{array}$ & $\begin{array}{c}\text { Energy } \\
\text { variational }\end{array}$ & $\begin{array}{c}\text { Energy } \\
\text { exact }\end{array}$ & $\begin{array}{c}E_{k e} \\
\text { exact }\end{array}$ & $\begin{array}{c}E_{\text {spin }} \\
\text { exact }\end{array}$ & $\begin{array}{c}\left\langle x^{2}\right\rangle^{1 / 2} \\
\text { exact }\end{array}$ \\
\hline 1 & 4.435 & -0.087 & -0.088 & -0.017 & 0.105 & 1.379 \\
2 & 2.054 & -0.174 & -0.192 & -0.469 & 0.277 & 0.891 \\
5 & 0.201 & -0.435 & -0.504 & -1.009 & 0.505 & 0.866 \\
10 & -0.614 & -0.885 & -0.889 & -1.472 & 0.582 & 0.895 \\
100 & -1.701 & -1.696 & -1.751 & -1.930 & 0.179 & 1.954 \\
\hline \hline
\end{tabular}

ergy Eq. (5) with respect to $\lambda$ and $\eta$. Results are shown in Table I.

Note incidentally that Mott's description of the polaron discussed earlier is also variational, corresponding to the trial wave function: $\psi(x)=N \cos [\pi x /(2 R)]$ for $x<R$ and 0 for $x$ $>R$ and $\chi(x)=\pi$ for $x>R$ and 0 for $x<R$, where the polaron radius $R$ is the variational parameter and $N$ is the normalization constant. Unlike the Mott form, our variational wave function allows for a cant angle other than 0 or $\pi$, allowing a better description of the boundary region of the polaron, which is important in the narrow-band limit.

In Fig. 2, we show the wave function and the cant angle obtained from the variational calculation for $\alpha=20$, which are also compared to the Mott solution as well as the exact solution obtained from the Euler-Lagrange equations, which is described later. Notice the gradual turnover of the cant angle from ferromagnetic to antiferromagnetic alignment as well as the penetration of the electronic wave function into the antiferromagnetic region. The exact $\psi(x)$ is more or less free-particlelike near the central ferromagnetic region, i.e., similar to the Mott solution, with its tail extending into the antiferromagnetic region. The variational parameters $\lambda$ and $\eta$ for several values of $\alpha$ are listed in Table I.

\section{EXACT SOLUTION USING EULER-LAGRANGE METHOD}

While the variational wave function is quite useful and provides an analytical form of the solution, it is actually possible to solve the problem exactly albeit numerically, using methods of the calculus of variations. ${ }^{22}$ The appropriate Euler-Lagrange equations that minimizes the energy expression Eq. (5) are given by

$$
\frac{\partial F}{\partial y}-\frac{d}{d x} \frac{\partial F}{\partial \dot{y}}+\frac{d^{2}}{d x^{2}} \frac{\partial F}{\partial \ddot{y}}=0
$$

where $y=\psi$ or $\chi$. The function $F \equiv f(x, \psi, \dot{\psi}, \ddot{\psi}, \chi, \dot{\chi}, \ddot{\chi})$ $+\beta \psi^{2}$, where $f$ is the integrand appearing in the energy expression Eq. (5) and where the normalization condition $\int_{-\infty}^{\infty} \psi^{2}(x) d x=1$ has been enforced via the Lagrange multiplier $\beta$. Using Eq. (5), the Euler-Lagrange equations become

$$
(4 \psi+\ddot{\psi}) \cos \frac{\chi}{2}+\frac{d^{2}}{d x^{2}}\left(\psi \cos \frac{\chi}{2}\right)-2 \beta \psi / t=0,
$$

$$
\left\{\alpha \psi(\psi+\ddot{\psi} / 2)-2 \cos \frac{\chi}{2}\right\} \sin \frac{\chi}{2}=0 .
$$

These are the two coupled differential equations, which we have solved by the following iterative procedure: (a) Choose a guess cant-angle function $\chi(x)$; (b) solve the first differential equation Eq. (10) by the Numerov or any of the standard methods to obtain $\psi$, (c) with this $\psi$, obtain the cant-angle function $\chi(x)$ by inverting Eq. (11); and (d) iterate the above steps until the function $\chi$ has converged to the desired accuracy. The energy may then be obtained from the expression (5). Our convergence criterion for $\chi(x)$ was that the computed energy be converged to the desired accuracy.

The functions $\psi(x)$ and $\chi(x)$ obtained from the solution of the Euler-Lagrange equations are shown in Fig. 3 for several values of the patameter $\alpha$. As seen from the figure, the magnetic polaron can have two types of behaviors depending on whether the ferromagnetic core of the polaron is saturated or not. For large values $\alpha>\alpha_{c}$, the ferromagnetic core is fully saturated (type II), while for smaller values, the ferromagnetic core is only partially saturated (type I). ${ }^{7}$ The value of $\alpha_{c}$ is found to be about 7.5 from the exact results obtained from the solution of the Euler-Lagrange equations.

The energy obtained from the variational wave function (6) is good overall as compared to the exact energy in a large range of $\alpha$. However, the electronic part $\psi(x)$ is not a good description of the wave function, especially for large values of $\alpha$, which may be expected by an inspection of its $\exp ( \pm \lambda x)$-type functional form. In fact, as seen from Table II, Mott's simple-minded wave function gives a better energy

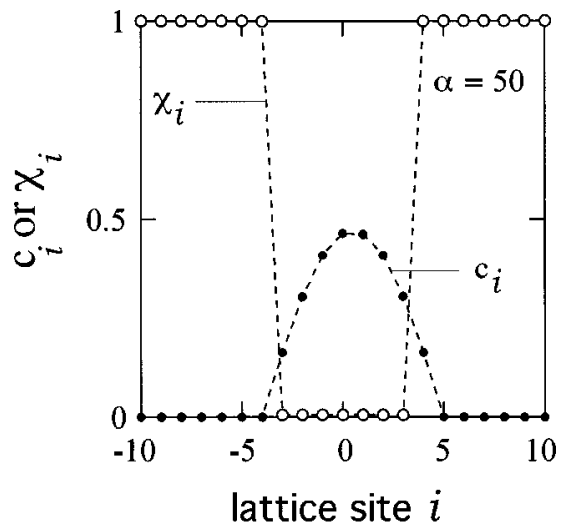

FIG. 4. The electronic wave function $c_{i}$ and the cant-angle function $\chi_{i}$ for the magnetic polaron in the discrete lattice case. The index $i$ denotes the lattice sites. Individual points are connected by dashed lines to guide the eye. Cant angles $\chi_{i}$ are in units of $\pi$. 


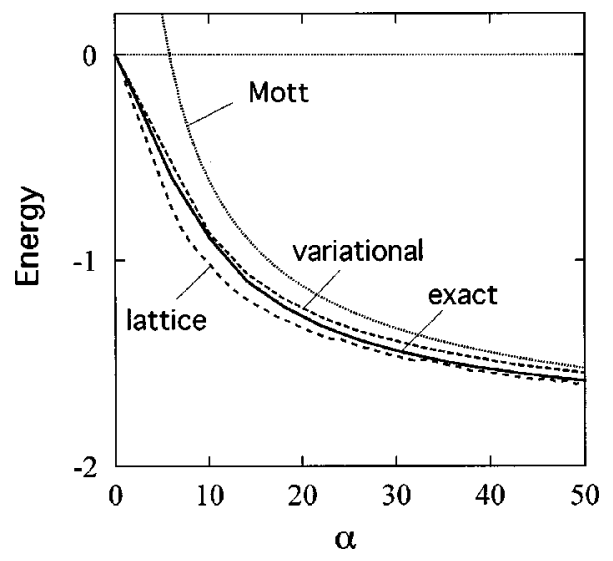

FIG. 5. Ground-state energy of the magnetic polaron as a function of the parameter $\alpha$. The exact energies are compared to the variational as well as the Mott results. The zero of energy being that of the propagating state in the antiferromagnetic lattice, our results indicate that the magnetic polaron forms a bound, self-trapped state for all values of $\alpha$. Energies for the discrete lattice case are also shown.

than the variational wave function for very large values of $\alpha$, e.g., for the case $\alpha=100$.

\section{DISCRETE LATTICE}

We have also solved the magnetic polaron problem for the discrete lattice by minimizing the energy expression Eq. (4) subject to the normalization condition for the electronic wave function. The relevant equations for the discrete lattice are given by

$$
\begin{gathered}
c_{n+1} \cos \left(\chi_{n} / 2\right)+c_{n-1} \cos \left(\chi_{n-1} / 2\right)+\lambda c_{n} / t=0, \\
4 \cos \left(\chi_{n} / 2\right)=\alpha\left(c_{n}^{*} c_{n+1}+c_{n} c_{n+1}^{*}\right),
\end{gathered}
$$

which were solved by an iterative procedure analogous to the procedure described earlier for the continuum case. The wave functions for the discrete lattice case are shown in Fig. 4 for the parameter $\alpha=50$.

The calculated energies for both the continuum and the lattice cases are shown in Fig. 5, where the energies obtained from the Euler-Lagrange method ("'exact") have been compared with the variational energies as well as with the Mott energies. Both the exact energies as well as the variational energies are lower than the Mott values (Table I). The variational solution is quite close to the exact results in the entire parameter range of $\alpha$. Unlike Mott's solution, our calculated energies are always negative with respect to the energy of the propagating state, implying that the magnetic polaron is selftrapped for all values of $\alpha$.

We have also shown in Fig. 5 the energy for the discrete lattice case, which is quite close to the energy obtained for the continuum case. This is remarkable considering the fact that the calculated polaron radius is only of the order of a few lattice constants. Even for such a small polaron size, the continuum approximation seems to work quite well.

The expectation value $\left\langle x^{2}\right\rangle^{1 / 2}$ as obtained from the exact results for the continuum case has been shown in Fig. 6. It is easy to see that in both the limits of $t \rightarrow 0$ or $\infty$, the electronic

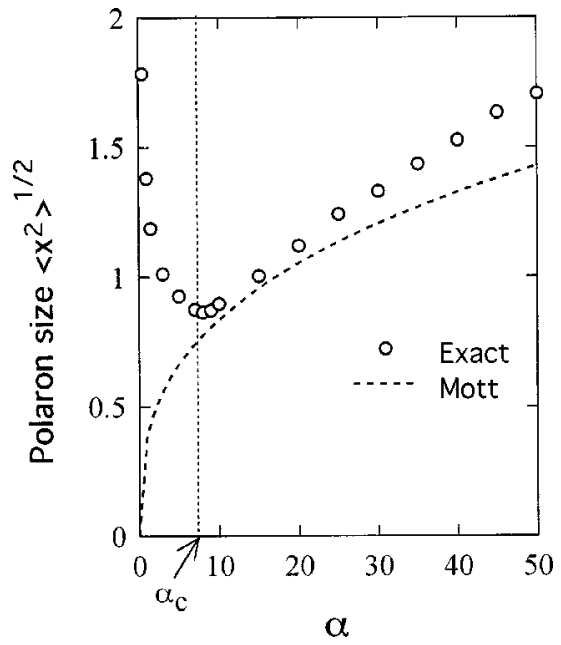

FIG. 6. Variation of $\left\langle x^{2}\right\rangle^{1 / 2}$ with $\alpha$ for the continuum case obtained from the exact calculation. The critical value $\alpha_{c} \approx 7.5$ separates the type-I and the type-II regions.

wave function is completely delocalized. In the latter case, the strong double exchange turns the entire lattice ferromagnetic and the electron gains the kinetic energy $-z t, z$ being the number of nearest neighbors. For $t \rightarrow 0$ the nearantiferromagnetic lattice is not able to produce a strong enough potential well to localize the electron. For $t=0$, the lattice is antiferromagnetic and the energy of the electron is in fact independent of its wave function, with the stipulation that at each lattice site, the electron spin is aligned along the direction of the localized spin at that lattice site.

\section{CONCLUSION}

In conclusion, we have solved a continuum model for the self-trapped magnetic polaron in an antiferromagnetic host lattice and obtained the exact solution by solving the coupled Euler-Lagrange differential equations. Solutions corresponding to type-I (unsaturated ferromagnetic core) and type-II (saturated ferromagnetic core) polarons were obtained for the parameter $\alpha<\alpha_{c}$ and $\alpha>\alpha_{c}\left(\alpha \equiv t / J S^{2}\right)$, respectively, with the critical value being $\alpha_{c} \simeq 7.5$ for the continuum model. A variational wave function, suitable to describe both the type-I and the type-II behaviors, was proposed and was found to reproduce the exact energy rather well. The polaron was found to be self-trapped for all values of $\alpha$, both in the continuum and the lattice models, if only the nearest-neighbor electron transfer is allowed. Higher-neighbor electron transfer, which permits electron transfer within the same magnetic sublattice, would tend to delocalize the polaronic wave function and so would the zero-point quantum fluctuations of the localized spins. Both these effects, which are not considered here, could in principle destabilize the self-trapped state for small values of $\alpha$.

\section{ACKNOWLEDGMENTS}

Part of the work was performed during a visit of one of the authors (S.S.) to the Institute of Theoretical Physics at the University of California, Santa Barbara, where it was supported in part by the National Science Foundation under Grant No. PHY94-07194. 
${ }^{1}$ P.-G. de Gennes, Phys. Rev. 118, 141 (1960).

${ }^{2}$ E.L. Nagaev, Zh. Éksp. Teor. Fiz. Pis'ma Red. 6, 484 (1967) [JETP Lett. 6, 18 (1967)].

${ }^{3}$ T. Kasuya, Solid State Commun. 8, 1635 (1970).

${ }^{4}$ M. Umehara and T. Kasuya, J. Phys. Soc. Jpn. 33, 602 (1972).

${ }^{5}$ N. F. Mott, Metal-Insulator Transitions (Taylor \& Francis, London, 1974).

${ }^{6}$ A. Mauger and D.L. Mills, Phys. Rev. Lett. 53, 1594 (1984).

${ }^{7}$ A. Mauger and D.L. Mills, Phys. Rev. B 31, 8024 (1985).

${ }^{8}$ E.L. Nagaev, Usp. Fiz. Nauk. 117, 437 (1975) [Sov. Phys. Usp. 18, 836 (1976)].

${ }^{9}$ S. von Molnar and S. Methfessel, J. Appl. Phys. 38, 959 (1967).

${ }^{10}$ P. Wachter, Solid State Commun. 8, 473 (1970).

${ }^{11}$ T. Kasuya, A. Yanase, and T. Takeda, Solid State Commun. 8, 1543 (1970).

${ }^{12}$ M. Umehara, Phys. Rev. B 54, 5523 (1996).
${ }^{13}$ E.L. Nagaev, J. Magn. Magn. Mater. 110, 39 (1992).

${ }^{14}$ J.M. De Teresa, M.R. Ibarra, P.A. Algarabel, C. Ritter, C. Marquina, J. Blasco, J. Garcia, A. del Moral, and Z. Arnold, Nature (London) 386, 256 (1997).

${ }^{15}$ M. Hennion, F. Moussa, J. Rodrigues-Carvajal, L. Pinsard, and A. Revcolevschi, Phys. Rev. B 56, R497 (1999).

${ }^{16}$ J.J. Neumeier and D.H. Goodwin, J. Appl. Phys. 85, 5591 (1999).

${ }^{17}$ C.M. Varma, Phys. Rev. B 54, 7328 (1996).

${ }^{18}$ E.L. Nagaev, Phys. Rev. B 60, 455 (1999).

${ }^{19}$ C.D. Batista, J. Eroles, M. Avignon, and B. Alascio, Phys. Rev. B 58, R14 689 (1998).

${ }^{20}$ S. Satpathy, Z.S. Popović, and F.R. Vukajlović, Phys. Rev. Lett. 76, 960 (1996); J. Appl. Phys. 79, 4555 (1996).

${ }^{21}$ P.W. Anderson and H. Hasegawa, Phys. Rev. 100, 675 (1955).

${ }^{22}$ R. Courant and D. Hilbert, Methods of Mathematical Physics (Interscience, New York, 1953), Vol. I. 